ESAIM: M2AN 46 (2012) 911-927

DOI: $10.1051 / \mathrm{m} 2 \mathrm{an} / 2011074$
ESAIM: Mathematical Modelling and Numerical Analysis

www.esaim-m2an.org

\title{
A MINIMUM EFFORT OPTIMAL CONTROL PROBLEM FOR ELLIPTIC PDES
}

\author{
Christian Clason $^{1}$, Kazufumi Ito ${ }^{2}$ and Karl Kunisch ${ }^{1}$
}

\begin{abstract}
This work is concerned with a class of minimum effort problems for partial differential equations, where the control cost is of $\mathrm{L}^{\infty}$-type. Since this problem is non-differentiable, a regularized functional is introduced that can be minimized by a superlinearly convergent semi-smooth Newton method. Uniqueness and convergence for the solutions to the regularized problem are addressed, and a continuation strategy based on a model function is proposed. Numerical examples for a convectiondiffusion equation illustrate the behavior of minimum effort controls.
\end{abstract}

Mathematics Subject Classification. 49J52, 49J20, 49K20.

Received February 2, 2011. Revised September 22, 2011.

Published online February 3, 2012.

\section{INTRODUCTION}

We investigate the optimal control problem

$$
\left\{\begin{array}{l}
\min _{u \in \mathrm{L}^{\infty}} \frac{1}{2}\|y-z\|_{\mathrm{L}^{2}}^{2}+\frac{\alpha}{2}\|u\|_{\mathrm{L}^{\infty}}^{2} \\
\text { s.t. } A y=u \quad \text { in } \Omega,
\end{array}\right.
$$

where $\alpha>0, \Omega$ is a bounded domain in $\mathbb{R}^{n}, A$ is a linear second order elliptic partial differential operator of convection-diffusion type carrying appropriate boundary conditions, and $z \in \mathrm{L}^{2}(\Omega)$. Problem (1.1) expresses the fact that we wish to determine the best possible control $u$ which steers the state $y$ as close as possible to $z$, with minimum effort. We consider (1.1) as a simple reference problem. The techniques to be presented here can certainly be generalized in many aspects. In particular, the results are applicable if the controls act on subdomains $\omega$ strictly contained in $\Omega$. We shall frequently consider an equivalent formulation given by

$$
\left\{\begin{array}{l}
\min _{c \in \mathbb{R}, u \in \mathrm{L}^{\infty}} \frac{1}{2}\|y-z\|_{\mathrm{L}^{2}}^{2}+\frac{\alpha}{2} c^{2} \\
\text { s.t. } A y=u \quad \text { in } \Omega, \\
\|u\|_{\mathrm{L}^{\infty}} \leq c,
\end{array}\right.
$$

\footnotetext{
Keywords and phrases. Optimal control, minimum effort, $\mathrm{L}^{\infty}$ control cost, semi-smooth Newton method.

1 Institute for Mathematics and Scientific Computing, University of Graz, Heinrichstrasse 36, 8010 Graz, Austria. christian.clason@uni-graz.at; karl.kunisch@uni-graz.at

2 Department of Mathematics, North Carolina State University, Raleigh, 27695-8205, North Carolina, USA.

kito@math.ncsu.edu
} 
where the nondifferentiability that appears in the cost of (1.1) is moved to the constraint set of (1.2). This problem resembles a bilaterally constrained optimal control problem, but it is different in that the bound on the control is itself a variable that is subject to minimization. Below, we shall consider yet another reformulation involving a scaling of the control according to $u \rightarrow c u$. This will have the advantage that the constraint is not parameter dependent but fixed, at the expense of a bilinear structure occurring in the transformed state-equation constraint.

Problems involving $\mathrm{L}^{\infty}$ control costs - so-called minimum effort problems - have received rather little attention in the mathematical literature so far despite their obvious practical relevance. This may be related to the obvious difficulty arising from the nondifferentiability appearing in the problem formulation. We shall demonstrate that semi-smooth Newton methods in a function space setting are an efficient method to overcome this difficulty. Published investigations of minimum effort problems focus on the case of - mostly linear - control systems in the context of ordinary differential equations. We particularly mention [10], where sufficient conditions for the optimal controls to be bang-bang are given. In [1], numerical approaches to solve minimum effort problems are discussed and applications to spacecraft maneuvers are given. The application of semi-smooth Newton methods to minimum effort problems is presented in [8]. In contrast, the corresponding problem for partial differential equations has been studied less frequently (e.g., in $[6,16]$ in the context of approximate and exact controllability of heat and wave equations). In passing we also point to a related but different class of problems, where instead of a bound on the controls, bounds on the state are minimized. This type of constraints can be interpreted as minimal invasion problems and was considered in $[2,5,11]$.

In Section 2 we discuss existence and uniqueness of a solution to (1.1), and present the first order optimality condition. Section 3 contains a regularization procedure that is the basis for the numerical treatment by a semi-smooth Newton method together with a continuation strategy based on a model function approach, all of which are investigated in Section 4. Numerical examples are presented in Section 5.

\section{Existence, Uniqueness, AND OPTIMALity SySTEM}

We first address well-posedness of the state equation. We consider the operator

$$
A y=-\sum_{j, k=1}^{n} \partial_{j}\left(a_{j k}(x) \partial_{k} y+d_{j}(x) y\right)+\sum_{j=1}^{n} b_{j}(x) \partial_{j} y+d(x) y,
$$

where the coefficients satisfy $a_{j k} \in \mathrm{C}^{0, \delta}(\bar{\Omega})$ for some $\delta \in(0,1)$ and $b_{j}, d \in \mathrm{L}^{\infty}(\Omega)$, and the corresponding Dirichlet problem

$$
\left\{\begin{aligned}
A y=g, & \text { in } \Omega, \\
y=0, & \text { on } \partial \Omega,
\end{aligned}\right.
$$

where the domain $\Omega \subset \mathbb{R}^{n}, n=2,3$, is open, bounded with at least Lipschitz continuous boundary $\partial \Omega$, and $g \in \mathrm{L}^{2}(\Omega)$ is given. If 0 is not an eigenvalue of $A$, this problem has a unique solution in $\mathrm{H}_{0}^{1}(\Omega)$. A sufficient assumption for this is the existence of constants $\lambda, \Lambda, \nu>0$ such that

$$
\left\{\begin{array}{l}
\lambda|\xi|_{2}^{2} \leq a_{j k} \xi_{j} \xi_{k} \quad \text { for all } \xi \in \mathbb{R}^{n}, \quad \sum_{j, k=1}^{n}\left|a_{j, k}\right|^{2} \leq \Lambda^{2}, \\
\lambda^{-2} \sum_{j=1}^{n}\left(\left|d_{j}\right|^{2}+\left|b_{j}\right|^{2}\right)+\lambda^{-1}|d| \leq \nu^{2}, \quad d-\partial_{j} d_{j} \geq 0, \quad \text { for all } 1 \leq j \leq n,
\end{array}\right.
$$

(cf., e.g., [4], Thm. 8.3). In particular, this implies the existence of a unique solution $y \in \mathrm{H}_{0}^{1}(\Omega)$ of the state equation $A y=u$ for any control $u \in \mathrm{L}^{\infty}(\Omega)$. We further assume that the domain $\Omega$ is sufficiently regular (e.g., $\partial \Omega$ is of class $C^{1,1}$ or $\Omega$ is a parallelepiped [9], pp. 169-189, [14], Thm. 2.24) that in addition $y \in \mathrm{H}^{2}(\Omega)$ holds. 
Consider now the minimum effort problem (1.2). Observe that (1.2) contains the implicit constraint $c \geq 0$. Except in the case $c^{*}=0$, problem (1.2) can equivalently be expressed by rescaling the control $u$ :

$$
\left\{\begin{array}{l}
\min _{c \in \mathbb{R}_{+}, u \in \mathrm{L}^{\infty}}\left\{J(y, c) \equiv \frac{1}{2}\|y-z\|_{\mathrm{L}^{2}}^{2}+\frac{\alpha}{2} c^{2}\right\} \\
\text { s.t. } A y=c u \quad \text { in } \Omega, \\
\|u\|_{\mathrm{L}^{\infty}} \leq 1 \quad \text { in } \Omega .
\end{array}\right.
$$

By standard arguments, we obtain existence of a minimizer $\left(y^{*}, u^{*}, c^{*}\right) \in \mathrm{H}_{0}^{1}(\Omega) \times \mathrm{L}^{\infty}(\Omega) \times \mathbb{R}_{+}$of $(\mathcal{P})$. For $c^{*}=0$, any control $u$ with $\|u\|_{L^{\infty}} \leq 1$ is a minimizer. The degenerate case $c^{*}=0$ can be excluded if and only if $J\left(y^{*}, c^{*}\right)<\frac{1}{2}\|z\|_{\mathrm{L}^{2}}^{2}$ with $A y^{*}=c^{*} u^{*}$ and $\left\|u^{*}\right\|_{\mathrm{L}^{\infty}} \leq 1$, which will henceforth be assumed.

For $c^{*} \neq 0$, this solution is unique. In fact, if $\left(c_{1}, u_{1}\right)$ and $\left(c_{2}, u_{2}\right)$ are two (possibly different) solutions to $(\mathcal{P})$ with $c_{1} \neq 0$ and $c_{2} \neq 0$, then they are also solutions to (1.1), where the cost can be expressed as $F(u)=\frac{1}{2} \| A^{-1} u-$ $z\left\|_{\mathrm{L}^{2}}^{2}+\frac{\alpha}{2}\right\| u \|_{\mathrm{L}^{\infty}}^{2}$. Since $A^{-1}$ is injective, $u \mapsto \frac{1}{2}\left\|A^{-1} u-z\right\|_{\mathrm{L}^{2}}^{2}$ is strictly convex. Furthermore, $u \mapsto \frac{\alpha}{2}\|u\|_{\mathrm{L}^{\infty}}^{2}$ is convex. Consequently, $u \mapsto F(u)$ is strictly convex on $\mathrm{L}^{\infty}(\Omega)$ and hence $u_{1}=u_{2}$ and consequently $c_{1}=c_{2}$ holds.

Using standard subdifferential calculus (cf., e.g., [3]), we obtain the existence of a Lagrange multiplier $p^{*} \in$ $\mathrm{H}_{0}^{1}(\Omega)$ satisfying the necessary optimality conditions

$$
\left\{\begin{array}{l}
\left\langle-p^{*}, u-u^{*}\right\rangle_{L^{2}} \geq 0 \quad \text { for all } u \text { with }\|u\|_{L^{\infty}} \leq 1 \\
\alpha c^{*}-\left\langle u^{*}, p^{*}\right\rangle_{L^{2}}=0 \\
y^{*}-z+A^{*} p^{*}=0 \\
A y^{*}-c^{*} u^{*}=0
\end{array}\right.
$$

From the assumption on the regularity of $\Omega$, we have in addition $p^{*} \in \mathrm{H}^{2}(\Omega)$.

By pointwise inspection of the first relation of (OS) we deduce that

$$
u(x)= \begin{cases}1 & \text { if } p(x)>0, \\ -1 & \text { if } p(x)<0, \\ t \in[-1,1] & \text { if } p(x)=0\end{cases}
$$

holds, which can be equivalently expressed as $u=\operatorname{sign}(p)$. Inserting this into the second relation of (OS) and eliminating $y$ and $u$ from the last two relations, we obtain the reduced optimality system

$$
\left\{\begin{array}{l}
A A^{*} p^{*}+c^{*} \operatorname{sign}\left(p^{*}\right)=A z, \\
\alpha c^{*}-\left\|p^{*}\right\|_{L^{1}}=0
\end{array}\right.
$$

where the first equation should be interpreted in variational form, i.e., as

$$
\left\langle A^{*} p^{*}, A^{*} v\right\rangle_{\mathrm{L}^{2}}+c^{*}\left\langle\operatorname{sign}\left(p^{*}\right), v\right\rangle_{\mathrm{L}^{2}}=\left\langle z, A^{*} v\right\rangle_{\mathrm{L}^{2}}
$$

for all $v \in \mathrm{H}_{0}^{1}(\Omega) \cap \mathrm{H}^{2}(\Omega)$.

\section{REGULARIZED PROBLEM}

From (OS'), it is clear that the optimality system is not differentiable even in a generalized sense. We therefore introduce the following regularization in Problem $(\mathcal{P})$, where we again only consider $c \geq 0$ :

$$
\left\{\begin{array}{l}
\min _{c \in \mathbb{R}_{+}, u \in \mathrm{L}^{\infty}}\left\{J_{\beta}(y, u, c) \equiv \frac{1}{2}\|y-z\|_{\mathrm{L}^{2}}^{2}+\frac{\beta c}{2}\|u\|_{\mathrm{L}^{2}}^{2}+\frac{\alpha}{2} c^{2}\right\} \\
\text { s.t. } A y=c u \quad \text { in } \Omega, \\
\|u\|_{\mathrm{L}^{\infty}} \leq 1 .
\end{array}\right.
$$


As before, existence of a minimizer $\left(y_{\beta}, u_{\beta}, c_{\beta}\right) \in \mathrm{H}_{0}^{1}(\Omega) \times \mathrm{L}^{\infty}(\Omega) \times \mathbb{R}_{+}$follows from standard arguments. The case $c_{\beta}=0$ is excluded by the assumption that $J\left(y^{*}, c^{*}\right)<\frac{1}{2}\|z\|_{L^{2}}^{2}$, where $\left(y^{*}, u^{*}, c^{*}\right)$ is the solution to $(\mathcal{P})$. In fact, if the $c_{\beta}$-component of the solution to $\left(\mathcal{P}_{\beta}\right)$ is zero, then

$$
\begin{aligned}
\frac{1}{2}\|z\|_{\mathrm{L}^{2}}^{2} & =\frac{1}{2}\left\|y_{\beta}-z\right\|_{\mathrm{L}^{2}}^{2}+\frac{\beta c_{\beta}}{2}\left\|u_{\beta}\right\|_{\mathrm{L}^{2}}^{2}+\frac{\alpha}{2} c_{\beta}^{2} \\
& \leq \frac{1}{2}\left\|y^{*}-z\right\|_{\mathrm{L}^{2}}^{2}+\frac{\beta c^{*}}{2}\left\|u^{*}\right\|_{\mathrm{L}^{2}}^{2}+\frac{\alpha}{2}\left(c^{*}\right)^{2}=J\left(y^{*}, c^{*}\right)<\frac{1}{2}\|z\|_{\mathrm{L}^{2}}^{2},
\end{aligned}
$$

which gives a contradiction.

Due to the bilinear structure of the equality constraint in $\left(\mathcal{P}_{\beta}\right)$, uniqueness of the solution is not obvious. The (technical) proof of the following statement is given in Appendix A.

Proposition 3.1. If $\alpha>0$ is sufficiently large, then the solution $\left(y_{\beta}, u_{\beta}, c_{\beta}\right)$ to $\left(\mathcal{P}_{\beta}\right)$ is unique for every $\beta>0$. For any $\alpha>0$ and given $c_{\beta}$, the corresponding components $u_{\beta}, y_{\beta}$ are unique, and conversely $c_{\beta}$ and hence $y_{\beta}$ is uniquely determined by $u_{\beta}$.

For $c_{\beta}>0$, we obtain the existence of a $p_{\beta} \in \mathrm{H}_{0}^{1}(\Omega)$ satisfying the necessary optimality conditions for $\left(\mathcal{P}_{\beta}\right)$ :

$$
\left\{\begin{array}{l}
\left\langle\beta u_{\beta}-p_{\beta}, u-u_{\beta}\right\rangle_{L^{2}} \geq 0 \quad \text { for all } u \text { with }\|u\|_{L^{\infty}} \leq 1 \\
\alpha c_{\beta}+\frac{\beta}{2}\left\|u_{\beta}\right\|_{L^{2}}^{2}-\left\langle u_{\beta}, p_{\beta}\right\rangle_{L^{2}}=0 \\
y_{\beta}-z+A^{*} p_{\beta}=0 \\
A y_{\beta}-c_{\beta} u_{\beta}=0
\end{array}\right.
$$

We have again by pointwise inspection of the first relation that

$$
u(x)=\operatorname{sign}_{\beta}(p)(x):= \begin{cases}1 & \text { if } p(x)>\beta, \\ -1 & \text { if } p(x)<-\beta, \\ \frac{1}{\beta} p(x) & \text { if }|p(x)| \leq \beta\end{cases}
$$

holds. Using again the higher regularity $p_{\beta} \in \mathrm{H}^{2}(\Omega)$, we can insert this into the second relation of $\left(\operatorname{OS}_{\beta}\right)$ and eliminate $y$ and $u$ to obtain

$$
\left\{\begin{array}{l}
A A^{*} p_{\beta}+c_{\beta} \operatorname{sign}_{\beta}\left(p_{\beta}\right)=A z \\
\alpha c_{\beta}-\left\|p_{\beta}\right\|_{L_{\beta}^{1}}=0
\end{array}\right.
$$

where we have defined

$$
\|p\|_{\mathrm{L}_{\beta}^{1}}:=\int_{\Omega}|p(x)|_{\beta} \mathrm{d} x, \quad|p(x)|_{\beta}:= \begin{cases}p(x)-\frac{\beta}{2} & \text { if } p(x)>\beta \\ -p(x)-\frac{\beta}{2} & \text { if } p(x)<-\beta, \\ \frac{1}{2 \beta} p(x)^{2} & \text { if }|p(x)| \leq \beta .\end{cases}
$$

Remark 3.2. The chosen regularization is motivated by the following consideration: We can write (1.2) in the form

$$
\min _{c}\left(\min _{u} \frac{1}{2}\left\|A^{-1} u-z\right\|_{\mathrm{L}^{2}}^{2}+I_{\left\{\|v\|_{L^{\infty}} \leq c\right\}}(u)\right)+\frac{\alpha}{2} c^{2},
$$

Formally applying Fenchel duality for the inner minimization problem (where we consider $c>0$ fixed), we obtain by noting that the Fenchel dual of the indicator function of the $\mathrm{L}^{\infty}$-ball is the scaled $\mathrm{L}^{1}$ norm

$$
\min _{c}\left(\sup _{p}-\frac{1}{2}\left\|A^{*} p+z\right\|_{L^{2}}^{2}-c\|p\|_{L^{1}}\right)+\frac{\alpha}{2} c^{2} .
$$


Our regularization now amounts to replacing the non-differentiable $\mathrm{L}^{1}$-norm by the quadratic approximation $\|p\|_{\mathrm{L}_{\beta}^{1}}$, which has second (Newton-)derivatives and can be considered as a Huber-type smoothing of the $\mathrm{L}^{1}$-norm. The optimality system for the regularized dual problem (after replacing $p$ by $-p$ ) is then given by $\left(\operatorname{OS}_{\beta}^{\prime}\right.$ ). In Appendix 6, we compare different regularization strategies, which will turn out to be less convenient.

Remark 3.3. The proposed approach can also be applied when the control acts on a proper subdomain $\omega \subset \Omega$. Introducing the extension operator $E_{\omega}$ from $\omega$ to $\Omega$, we consider the regularized problem

$$
\left\{\begin{array}{l}
\min _{c \in \mathbb{R}_{+}, u \in \mathrm{L}^{2}(\omega)} \frac{1}{2}\|y-z\|_{\mathrm{L}^{2}}^{2}+\frac{\beta c}{2}\|u\|_{\mathrm{L}^{2}(\omega)}^{2}+\frac{\alpha}{2} c^{2} \\
\text { s.t. } A y=c E_{\omega} u \quad \text { in } \Omega \\
\|u\|_{\mathrm{L}^{\infty}(\omega)} \leq 1
\end{array}\right.
$$

with the necessary optimality conditions

$$
\begin{cases}c\left\langle\beta u-E_{\omega}^{*} p, \tilde{u}-u\right\rangle_{\mathrm{L}^{2}(\omega)} \geq 0 & \text { for all } \tilde{u} \text { with }\|\tilde{u}\|_{\mathrm{L}^{\infty}(\omega)} \leq 1, \\ \alpha c+\frac{\beta}{2}\|u\|_{\mathrm{L}^{2}(\omega)}^{2}-\langle u, p\rangle_{\mathrm{L}^{2}(\omega)}=0 & \text { if } c>0, \\ y-z+A^{*} p=0 & \\ A y-c E_{\omega} u=0, & \end{cases}
$$

where $E_{\omega}^{*}$ denotes the restriction operator to $\omega$. By case discrimination and pointwise inspection we can again obtain the reduced optimality system

$$
\left\{\begin{array}{l}
A A^{*} p+c \operatorname{sign}_{\beta}\left(\chi_{\omega} p\right)=A z, \\
\alpha c-\left\|\chi_{\omega} p\right\|_{\mathrm{L}_{\beta}^{1}}=0
\end{array}\right.
$$

where $\chi_{\omega}=E_{\omega} E_{\omega}^{*}$ is the characteristic function of $\omega$. The solution to this system can be computed using the semi-smooth Newton method described in Section 4.1 after changing the definition of the active and inactive sets to $\mathcal{A}_{+} \cap \omega, \mathcal{A}_{-} \cap \omega$ and $\mathcal{I} \cap \omega$, respectively.

We next address the convergence of solutions to $\left(\mathcal{P}_{\beta}\right)$ as $\beta \rightarrow 0$. First, we show monotonicity properties of the solutions with respect to $\beta$.

Lemma 3.4. For $\beta>0$ let $\left(y_{\beta}, u_{\beta}, c_{\beta}\right)$ denote any solution to $\left(\mathcal{P}_{\beta}\right)$ and let $\left(y^{*}, u^{*}, c^{*}\right)$ denote the solution to $(\mathcal{P})$. Then for any $\beta<\beta^{\prime}$ we have that

$$
\begin{aligned}
c_{\beta^{\prime}}\left\|u_{\beta^{\prime}}\right\|_{\mathrm{L}^{2}}^{2} & \leq c_{\beta}\left\|u_{\beta}\right\|_{\mathrm{L}^{2}}^{2}, \\
J\left(y_{\beta}, c_{\beta}\right) & \leq J\left(y_{\beta^{\prime}}, c_{\beta^{\prime}}\right), \\
J\left(y_{\beta}, c_{\beta}\right)+\frac{\beta c_{\beta}}{2}\left\|u_{\beta}\right\|_{\mathrm{L}^{2}}^{2} & \leq J\left(y^{*}, c^{*}\right)+\frac{\beta c^{*}}{2}\left\|u^{*}\right\|_{\mathrm{L}^{2}}^{2} .
\end{aligned}
$$

Proof. For $0 \leq \beta<\beta^{\prime}$ (where $c_{0} \equiv c^{*}$ etc.) we have that

$$
J\left(y_{\beta}, c_{\beta}\right)+\frac{\beta c_{\beta}}{2}\left\|u_{\beta}\right\|_{\mathrm{L}^{2}}^{2} \leq J\left(y_{\beta^{\prime}}, c_{\beta^{\prime}}\right)+\frac{\beta c_{\beta^{\prime}}}{2}\left\|u_{\beta^{\prime}}\right\|_{\mathrm{L}^{2}}^{2}
$$

which implies

$$
\begin{aligned}
J\left(y_{\beta}, c_{\beta}\right)+\frac{\beta c_{\beta}}{2}\left\|u_{\beta}\right\|_{\mathrm{L}^{2}}^{2}+\frac{\left(\beta^{\prime}-\beta\right) c_{\beta^{\prime}}}{2}\left\|u_{\beta^{\prime}}\right\|_{\mathrm{L}^{2}}^{2} & \leq J\left(y_{\beta^{\prime}}, c_{\beta^{\prime}}\right)+\frac{\beta^{\prime} c_{\beta^{\prime}}}{2}\left\|u_{\beta^{\prime}}\right\|_{\mathrm{L}^{2}}^{2} \\
& \leq J\left(y_{\beta}, c_{\beta}\right)+\frac{\beta^{\prime} c_{\beta}}{2}\left\|u_{\beta}\right\|_{\mathrm{L}^{2}}^{2}
\end{aligned}
$$


From the outer inequalities we deduce that

$$
\left(\beta-\beta^{\prime}\right)\left(c_{\beta^{\prime}}\left\|u_{\beta^{\prime}}\right\|_{\mathrm{L}^{2}}^{2}-c_{\beta}\left\|u_{\beta}\right\|_{\mathrm{L}^{2}}^{2}\right) \leq 0
$$

which implies relation (3.1).

From the first inequality of (3.4), we obtain

$$
J\left(y_{\beta}, c_{\beta}\right)-J\left(y_{\beta^{\prime}}, c_{\beta^{\prime}}\right) \leq \beta\left(c_{\beta}\left\|u_{\beta}\right\|_{\mathrm{L}^{2}}^{2}-c_{\beta^{\prime}}\left\|u_{\beta^{\prime}}\right\|_{\mathrm{L}^{2}}^{2}\right) \leq 0,
$$

and relation (3.2) follows.

Finally, relation (3.3) is a consequence of the second inequality of (3.4) by setting $\beta=0$ and $\beta^{\prime}=\beta$.

We can now show strong subsequential convergence of minimizers of $\left(\mathcal{P}_{\beta}\right)$.

Proposition 3.5. Any selection of solutions $\left\{\left(y_{\beta}, u_{\beta}, c_{\beta}\right)\right\}_{\beta>0}$ of $\left(\mathcal{P}_{\beta}\right)$ is bounded in $\mathrm{H}_{0}^{1}(\Omega) \times \mathrm{L}^{\infty}(\Omega) \times \mathbb{R}_{+}$. For $\beta \rightarrow 0$, it converges weak $-\star$ to the solution to $(\mathcal{P})$, and the convergence is strong in $\mathrm{H}_{0}^{1}(\Omega) \times \mathrm{L}^{q}(\Omega) \times \mathbb{R}_{+}$ for any $q \in[1, \infty)$.

Proof. Since $(y, u, c)=(0,0,0)$ is feasible for the constraints in $\left(\mathcal{P}_{\beta}\right)$, we have

$$
\left\|y_{\beta}-z\right\|_{\mathrm{L}^{2}}^{2}+\beta c_{\beta}\left\|u_{\beta}\right\|_{\mathrm{L}^{2}}^{2}+\alpha c_{\beta}^{2} \leq\|z\|_{\mathrm{L}^{2}}^{2}
$$

and hence $\left\{c_{\beta}\right\}_{\beta>0}$ is bounded. The family $\left\{u_{\beta}\right\}_{\beta>0}$ is bounded by 1 in $L^{\infty}(\Omega)$ and consequently $\left\{y_{\beta}\right\}_{\beta>0}$ is bounded in $\mathrm{H}_{0}^{1}(\Omega)$ for any $q \in[1, \infty)$.

Hence there exists $(\bar{y}, \bar{u}, \bar{c}) \in \mathrm{H}_{0}^{1}(\Omega) \times \mathrm{L}^{\infty}(\Omega) \times \mathbb{R}_{+}$such that, on a subsequence denoted by the same symbols, $\left(y_{\beta}, u_{\beta}, c_{\beta}\right) \rightarrow^{\star}(\bar{y}, \bar{u}, \bar{c})$ holds in $\mathrm{H}_{0}^{1}(\Omega) \times \mathrm{L}^{\infty}(\Omega) \times \mathbb{R}$. Passing to the limit in the variational formulation of $A y_{\beta}=c_{\beta} u_{\beta}$, we find that $A \bar{y}=\overline{c u}$. By the weak lower semicontinuity of the $\mathrm{L}^{\infty}$-norm, we have that $\|\bar{u}\|_{L^{\infty}} \leq 1$. Weak lower semicontinuity of $J_{\beta}$ from $\mathrm{L}^{2}(\Omega) \times \mathrm{L}^{2}(\Omega) \times \mathbb{R}_{+} \rightarrow \mathbb{R}$ implies that $(\bar{y}, \bar{u}, \bar{c})$ is a solution to $(\mathcal{P})$. Since the solution to $(\mathcal{P})$ is unique, $(\bar{y}, \bar{u}, \bar{c})$ coincides with $\left(y^{*}, u^{*}, c^{*}\right)$ and the whole family $\left\{\left(y_{\beta}, u_{\beta}, c_{\beta}\right)\right\}_{\beta>0}$ converges.

To show strong convergence, we insert the weak limit $\left(u^{*}, c^{*}\right)$ in the inequality (3.1) (setting $\left.\beta=0\right)$ to deduce from the lower semicontinuity of the norm that

$$
c_{\beta}\left\|u_{\beta}\right\|_{\mathrm{L}^{2}}^{2} \leq c^{*}\left\|u^{*}\right\|_{\mathrm{L}^{2}}^{2} \leq \liminf _{\beta \rightarrow 0} c_{\beta}\left\|u_{\beta}\right\|_{\mathrm{L}^{2}}^{2}
$$

holds. From this, we deduce

$$
\limsup _{\beta \rightarrow 0}\left\|u_{\beta}\right\|_{\mathrm{L}^{2}}^{2} \leq\left\|u^{*}\right\|_{\mathrm{L}^{2}}^{2} \leq \liminf _{\beta \rightarrow 0}\left\|u_{\beta}\right\|_{\mathrm{L}^{2}}^{2}
$$

and hence strong convergence in $\mathrm{L}^{2}(\Omega)$ - and thus in $\mathrm{L}^{q}(\Omega)$ for every $q \in[1, \infty)$ as well - of the subsequence $u_{\beta}$ to $u^{*}$. This also implies the strong convergence of $y_{\beta}=A^{-1}\left(c_{\beta} u_{\beta}\right)$ in $\mathrm{H}_{0}^{1}(\Omega)$.

Inserting $\left(y^{*}, u^{*}, c^{*}\right)$ into $(3.3)$ (setting $\left.\beta=0\right)$ yields

$$
0 \leq J\left(y_{\beta}, c_{\beta}\right)-J\left(y^{*}, c^{*}\right) \leq \beta\left(c^{*}\left\|u^{*}\right\|_{\mathrm{L}^{2}}-c_{\beta}\left\|u_{\beta}\right\|_{\mathrm{L}^{2}}\right) .
$$

From the strong convergence of $u_{\beta}$, we therefore obtain the following convergence rate result.

Corollary 3.6. As $\beta \rightarrow 0$, it holds that

$$
J\left(y_{\beta}, c_{\beta}\right)-J\left(y^{*}, c^{*}\right)=o(\beta) .
$$




\section{Solution of optimality System}

In this section, we discuss the computation of approximate minimizers of $(\mathcal{P})$. The first subsection is concerned with the solution for fixed $\beta>0$ of the regularized optimality system $\left(\mathrm{OS}_{\beta}\right)$. We then propose a continuation strategy in $\beta$ where the stopping criterion is based on a model function.

\subsection{Semi-smooth Newton method}

For the numerical solution of the regularized problem $\left(\mathcal{P}_{\beta}\right)$, we consider the reduced optimality system $\left(\mathrm{OS}_{\beta}^{\prime}\right)$ as an operator equation $T(p, c)=(0,0)$ for

$$
T: \mathrm{H}_{0}^{2}(\Omega) \times \mathbb{R}_{+} \rightarrow \mathrm{H}^{-2}(\Omega) \times \mathbb{R}, \quad(p, c) \mapsto\left(\begin{array}{c}
A A^{*} p+c \operatorname{sign}_{\beta}(p)-A z \\
\alpha c-\|p\|_{\mathrm{L}_{\beta}^{1}}
\end{array}\right),
$$

where $\mathrm{H}_{0}^{2}(\Omega):=\mathrm{H}_{0}^{1}(\Omega) \cap \mathrm{H}^{2}(\Omega)$ and $\mathrm{H}^{-2}(\Omega):=\left(\mathrm{H}_{0}^{2}(\Omega)\right)^{*}$. Obviously, $T$ is differentiable with respect to $c$. We next argue Newton differentiability of $T$ with respect to $p$. First observe that

$$
\operatorname{sign}_{\beta}(v)=\frac{1}{\beta}(v-\max (0, v-\beta)-\min (0, v+\beta))
$$

and recall (e.g., from [7], Thm. 8.5; see also [12]) that for any $\beta \in \mathbb{R}$, the function $z \mapsto \max (0, z-\beta)$ is Newton differentiable from $\mathrm{L}^{p}(\Omega)$ to $\mathrm{L}^{q}(\Omega)$ for any $p>q \geq 1$ with its Newton derivative in direction $h$ given pointwise by

$$
\left(D_{N} \max (0, v-\beta) h\right)(x)= \begin{cases}h(x), & \text { if } v(x)>\beta, \\ 0, & \text { if } v(x) \leq \beta\end{cases}
$$

An analogous statement holds for the min function. The function $\operatorname{sign}_{\beta}$ is thus Newton differentiable from $\mathrm{L}^{p}(\Omega)$ to $\mathrm{L}^{q}(\Omega)$ as well, where the Newton derivative of $\operatorname{sign}_{\beta}$ is given by

$$
\left(D_{N} \operatorname{sign}_{\beta}(p) h\right)(x)= \begin{cases}0, & \text { if }|p(x)|>\beta \\ \frac{1}{\beta} h(x), & \text { if }|p(x)| \leq \beta\end{cases}
$$

Since the mapping $\psi: \mathbb{R} \rightarrow \mathbb{R}, t \mapsto|t|_{\beta}$, is differentiable with globally Lipschitz continuous derivative $t \mapsto \operatorname{sign}_{\beta}(t), \psi$ defines a differentiable Nemytskii operator from $L^{p}(\Omega)$ to $L^{2}(\Omega)$ for every $p \geq 4$ (see, e.g., [15], Chap. 4.3, and the references therein). This yields the Newton differentiability of $\|\cdot\|_{L_{\beta}^{1}}$ from $L^{p}(\Omega), p \geq 4$, to $\mathbb{R}$, with Newton derivative

$$
D_{N}\left(\|p\|_{\mathrm{L}_{\beta}^{1}}\right) h=\left\langle\operatorname{sign}_{\beta}(p), h\right\rangle_{\mathrm{L}^{2}} .
$$

The Newton differentiability of $T$ thus follows from the smoothing properties of $A A^{*}$.

Defining the active and inactive sets by

$$
\begin{aligned}
\mathcal{A}_{+} & =\left\{x \in \Omega: p^{k}(x)>\beta\right\}, \\
\mathcal{A}_{-} & =\left\{x \in \Omega: p^{k}(x)<-\beta\right\}, \\
\mathcal{A} & =\mathcal{A}_{+} \cup \mathcal{A}_{-}, \quad \mathcal{I}=\Omega \backslash \mathcal{A}
\end{aligned}
$$

with indicator functions $\chi_{\mathcal{A}_{+}}, \ldots, \chi_{\mathcal{I}}$, a semi-smooth Newton step consists in finding $\delta p, \delta c$ for given $p^{k}, c^{k}$ such that

$$
\left\{\begin{array}{l}
A A^{*} \delta p+c^{k} \frac{1}{\beta} \chi_{\mathcal{I}} \delta p+\operatorname{sign}_{\beta}\left(p^{k}\right) \delta c=-\left(A A^{*} p^{k}+c \operatorname{sign}_{\beta}\left(p^{k}\right)-A z\right), \\
\alpha \delta c-\left\langle\operatorname{sign}_{\beta}\left(p^{k}\right), \delta p\right\rangle=-\left(\alpha c^{k}-\left\|p^{k}\right\|_{\mathrm{L}_{\beta}^{1}}\right)
\end{array}\right.
$$

holds.

We now show that the Newton system (4.1) is uniformly invertible for fixed $\beta>0$. 
Proposition 4.1. For each $(p, c) \in \mathrm{H}_{0}^{2}(\Omega) \times \mathbb{R}_{+}$, the mapping $M: \mathrm{H}_{0}^{2}(\Omega) \times \mathbb{R} \rightarrow \mathrm{H}^{-2}(\Omega) \times \mathbb{R}$,

$$
M(\delta p, \delta c):=\left(\begin{array}{c}
A A^{*} \delta p+c \frac{1}{\beta} \chi_{\mathcal{I}} \delta p+\operatorname{sign}_{\beta}(p) \delta c \\
\alpha \delta c-\left\langle\operatorname{sign}_{\beta}(p), \delta p\right\rangle
\end{array}\right)
$$

is invertible, and there exists a constant $C>0$ independent of $(p, c)$ such that there holds

$$
\left\|M^{-1}\right\| \leq C
$$

Proof. Due to the regularity of $\Omega$, we have that $A^{*}$ acts as an isomorphism from $\mathrm{H}_{0}^{2}(\Omega)$ to a closed subspace of $\mathrm{L}^{2}(\Omega)$. It thus suffices to observe that

$$
\langle(\delta p, \delta c), M(\delta p, \delta c)\rangle=\left\|A^{*} \delta p\right\|_{\mathrm{L}^{2}}^{2}+\frac{c}{\beta}\left\|\delta p \chi_{\mathcal{I}}\right\|_{\mathrm{L}^{2}}^{2}+\alpha \delta c^{2}>0
$$

for $(\delta p, \delta c) \neq 0$, independent of $p$ and $c>0$.

Thus, system (4.1) is semi-smooth, and from standard results (e.g., [7], Thm. 8.5) we deduce the following convergence result for the semi-smooth Newton method.

Theorem 4.2. For every $\alpha, \beta>0$, the Newton iteration (4.1) converges superlinearly to the solution $\left(p_{\beta}, c_{\beta}\right)$ of $\left(\mathrm{OS}_{\beta}^{\prime}\right)$, provided that $\left(p^{0}, c^{0}\right)$ is sufficiently close to $\left(p_{\beta}, c_{\beta}\right)$.

The following finite termination property (e.g., [7], Rem. 7.1.1) will be useful in formulating a continuation scheme in $\beta$ :

Proposition 4.3. If $\mathcal{A}_{+}^{k+1}=\mathcal{A}_{+}^{k}$ and $\mathcal{A}_{-}^{k+1}=\mathcal{A}_{-}^{k}$ holds, then $T\left(p^{k+1}, c^{k+1}\right)=0$.

\subsection{Continuation strategy for $\beta$}

While Theorem 4.2 guarantees locally superlinear convergence for every $\beta>0$, in practice the region of convergence for the semi-smooth Newton method shrinks with decreasing $\beta$. In order to compute a good approximation of the original minimum effort problem $(\mathcal{P})$, we make use of a continuation approach: Starting with large $\beta_{n}$, we compute the minimizer $\left(p_{\beta_{n}}, c_{\beta_{n}}\right)$, decrease $\beta_{n}$ by a given factor $q_{m}$ and compute the corresponding minimizers $\left(p_{\beta_{n+1}}, c_{\beta_{n+1}}\right)$ starting from the initial guess $\left(p^{0}, c^{0}\right)=\left(p_{\beta_{n}}, c_{\beta_{n}}\right)$. If the Newton iteration did not converge after a fixed number of iterations (as determined by the change in active sets), we increase the reduction factor by setting $q_{m+1}=\left(q_{m}\right)^{t}$ for a fixed $t<1$ and restart the iteration with new $\beta_{n}=q_{m+1} \beta_{n-1}$.

Let us address the optimal stopping of the decrease of the regularization parameter. For very small values of $\beta$ there is little change in the value of $c_{\beta}$ and $c_{\beta}\left\|u_{\beta}\right\|_{L^{2}}^{2}$. This observation from numerical tests can be used to develop a stopping rule based on a model function. From Lemma 3.4 it is known that $\beta \mapsto c_{\beta}\left\|u_{\beta}\right\|_{\mathrm{L}^{2}}^{2}$ is monotonically decreasing. Let $\mu>0$ denote the desired efficiency level of the regularization term. Then the stopping parameter $\hat{\beta}$ is chosen such that

$$
c_{\hat{\beta}}\left\|u_{\hat{\beta}}\right\|_{\mathrm{L}^{2}}^{2}>\mu c^{*}\left\|u^{*}\right\|_{\mathrm{L}^{2}}^{2} .
$$

Since $c^{*}$ and $u^{*}$ are unknown, we propose to introduce a model function $m(\beta)$ which approximates $\beta \mapsto c_{\beta}\left\|u_{\beta}\right\|_{\mathrm{L}^{2}}^{2}$. The specific choice we make is

$$
m(\beta)=\frac{K_{1}}{\left(K_{2}+\beta\right)^{2}} .
$$

Since $c^{*}\left\|u^{*}\right\|_{\mathrm{L}^{2}}^{2}$ is finite, we can expect that $0<K_{1}<\infty$ and $0<K_{2}<\infty$. The constants $K_{1}, K_{2}$ can be determined by interpolation from evaluations with two successive solutions to $\left(\mathcal{P}_{\beta}\right)$. The continuation is then stopped if

$$
c_{\beta_{n}}\left\|u_{\beta_{n}}\right\|_{\mathrm{L}^{2}}^{2}>\mu m_{n}(0)
$$




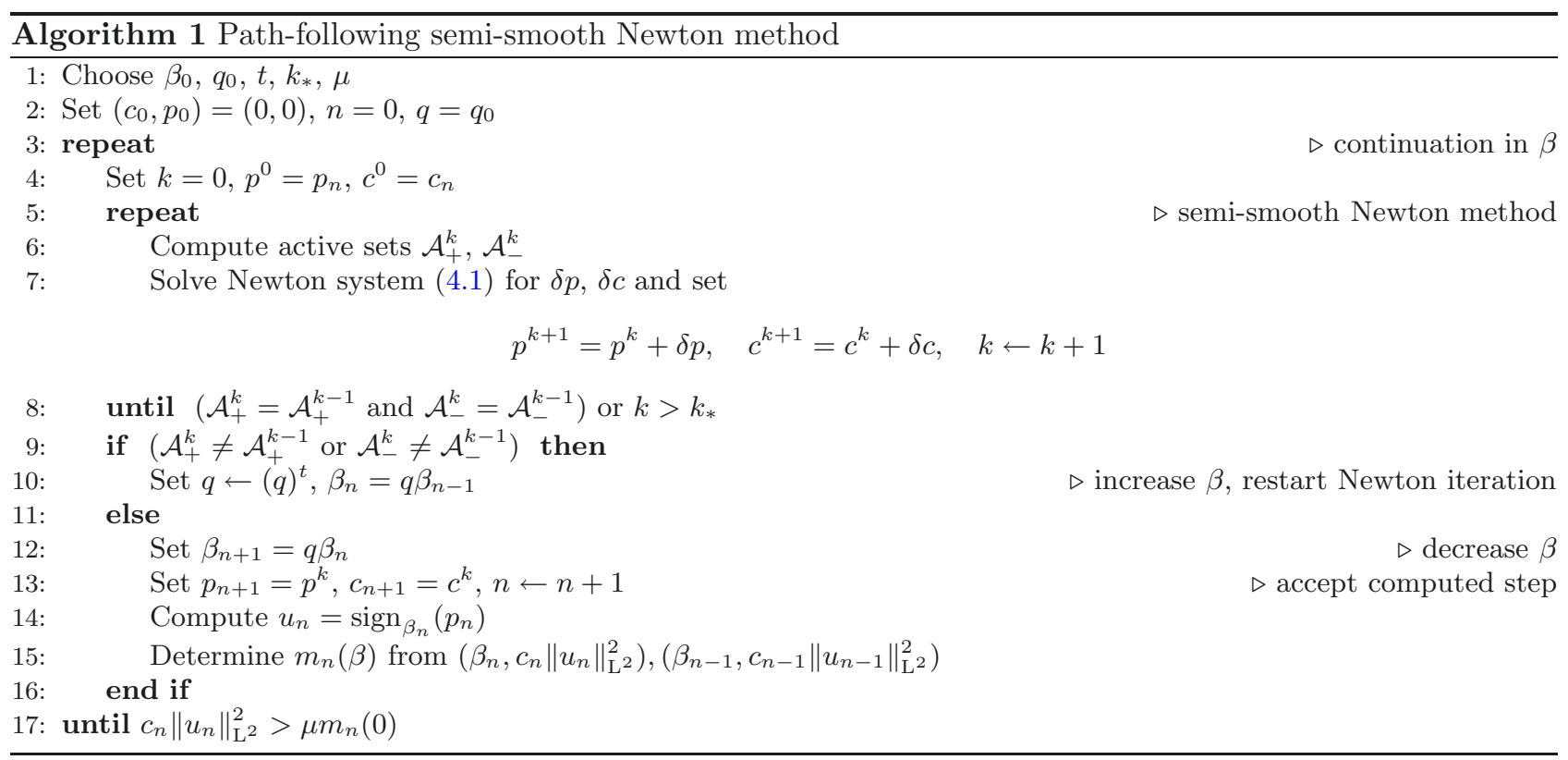

is satisfied, where $m_{n}$ is constructed from the interpolation conditions at $\beta_{n}$ and $\beta_{n-1}$.

The choice (4.2) for $m$ is based partly on numerical experience and partly on the following heuristic considerations. The necessary optimality condition implies that

$$
0=c A^{-1} u-z+\beta A u,
$$

where we ignore the inequality constraint on $u$. Considering $A$ as a scalar variable, rather than as an operator, and denoting it by $a$ henceforth, we have

$$
\left(c a^{-2}+\beta\right) u-a^{-1} z=0 .
$$

Here we may consider $u$ as the value of $u(x)$ at some $x$ where the constraint is not yet active. The range of interest for creating the model function covers small values of $\beta$, where numerical results show little dependence of $c_{\beta}$ on $\beta$. Assuming therefore that $c$ is a constant, and differentiating (4.3) with respect to $\beta$, we obtain

$$
\left(c a^{-2}+\beta\right) \frac{\mathrm{d}}{\mathrm{d} \beta} u+u=0 .
$$

The solution to this ordinary differential equation is given by $u=\frac{k_{1}}{c a^{-2}+\beta}$. This suggests using $m(\beta)$ as a model function for $c_{\beta}\left\|u_{\beta}\right\|_{\mathrm{L}^{2}}^{2}$.

The full procedure for the numerical approximation of the solution to $(\mathcal{P})$ is given as Algorithm 1 .

Remark 4.4. The convergence of the path-following method can be accelerated by starting with a damped Newton iteration ( $c f .[13])$, where we only take fractional Newton steps. In our experiments, a sequence of step sizes $\tau^{k}=\frac{k+1}{k+2}$ showed good results. While this modification is not necessary for the convergence of the method, it allows larger steps in the decrease of $\beta$. The benefit depends on $\alpha$, from about $20 \%$ performance increase for $\alpha=10^{-2}$ to about $80 \%$ for $\alpha=10^{-5}$. Since the focus of this work is not on optimal performance of the numerical solution, the examples shown below do not make use of this damping strategy. 


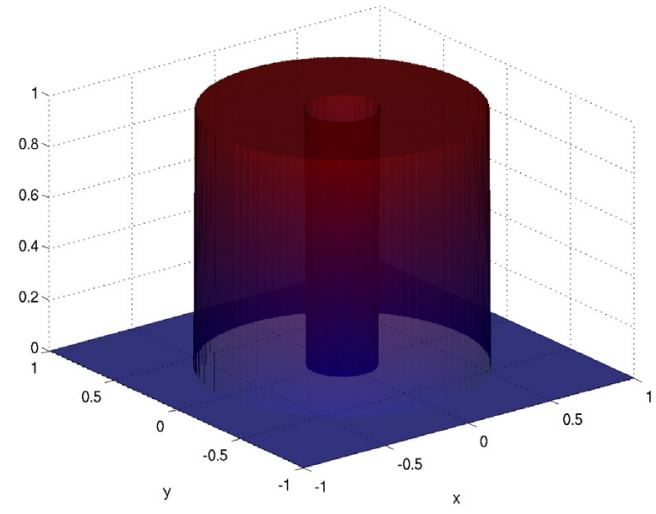

(A) $z_{1}$

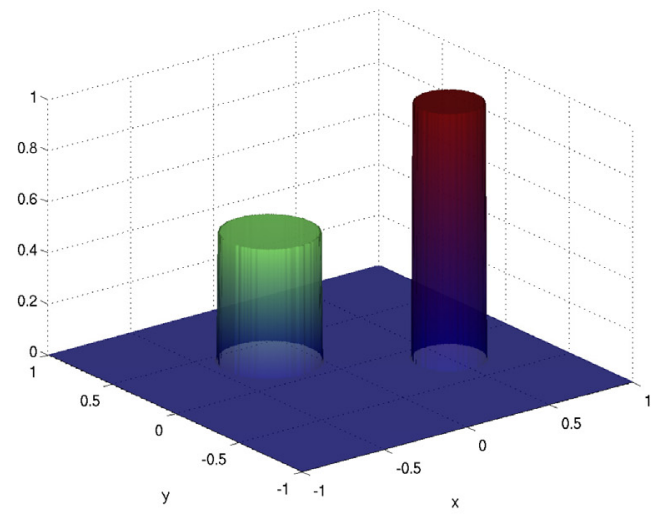

(в) $z_{2}$

Figure 1. Target functions.

TABLE 1. Optimal $\mathrm{L}^{\infty}$-bounds $c_{\alpha}$ for targets $z_{1}$ (left) and $z_{2}$ (right) and different $\alpha$.

\begin{tabular}{cccc}
\hline$\alpha$ & $5 \times 10^{-3}$ & $5 \times 10^{-4}$ & $5 \times 10^{-3}$ \\
\hline$c_{\alpha}$ & 1.9622 & 4.4236 & 9.8518 \\
\hline
\end{tabular}

\begin{tabular}{cccc}
\hline$\alpha$ & $5 \times 10^{-3}$ & $5 \times 10^{-4}$ & $5 \times 10^{-3}$ \\
\hline$c_{\alpha}$ & 0.8788 & 2.6066 & 6.8161 \\
\hline
\end{tabular}

\section{Numerical EXAMPLES}

To illustrate the features of the optimal controls arising in the minimum effort problem, we consider convective-diffusive transport, which is described by the operator $A y=-\nu \Delta y+b \cdot \nabla y$ with $\nu=0.1$ and $b=(-1,0)^{T}$ with homogeneous Dirichlet conditions on the unit square $[-1,1]^{2}$. We show results for two target functions

$$
\begin{aligned}
& z_{1}(x, y)=\chi_{\left\{x^{2}+y^{2}<1 / 2\right)} \chi_{\left\{x^{2}+y^{2}>1 / 32\right\}}, \\
& z_{2}(x, y)=\chi_{\left\{(x-0.5)^{2}+(y+0.2)^{2}<1 / 32\right\}}+\frac{1}{2} \chi_{\left\{(x+0.2)^{2}+(y-0.3)^{2}<1 / 16\right\}},
\end{aligned}
$$

which are shown in Figure 1.

The parameters in Algorithm 1 were set to $\beta_{0}=1, q_{0}=10^{-1}, t=0.5, k_{*}=10$, and $\mu=0.99$. The differential operators were discretized using finite differences on a uniform grid with $N=256$ nodes in each direction. A MATLAB function implementing Algorithm 1 can be downloaded from http://www. uni-graz.at/ clason.

We first compare the optimal (scaled) controls $(c u)_{\alpha} \equiv c^{*} u^{*}$ for different values of $\alpha$. The controls and corresponding states $y_{\alpha}$ are shown in Figure 2 for the target $z_{1}$ and in Figure 3 for the target $z_{2}$. The bang-bang nature of the minimum effort control can be seen clearly. The optimal $\mathrm{L}^{\infty}$ bounds $c_{\alpha}$ are given in Table 1 . In all cases, the optimal control $u_{\alpha}$ is feasible, i.e., $\max \left(u_{\alpha}\right)=-\min \left(u_{\alpha}\right)=1$. According to the model function, the continuation was stopped around $2 \times 10^{-7}$ in all cases except for target $z_{1}$ with $\alpha=5 \times 10^{-3}$, where the iteration was terminated at $2.4 \times 10^{-6}$.

We illustrate the convergence behavior with respect to $\beta$ exemplarily for the target $z_{2}$ and $\alpha=5 \times 10^{-3}$ in Figure 4. Figure $4 \mathrm{a}$ shows the iteration history of $c_{\beta}$, where every circle represents a computed value. Figure $4 \mathrm{~b}$ illustrates Corollary 3.6 by plotting the difference between the current functional value $J(\beta) \equiv J\left(y_{\beta}, c_{\beta}\right)$ and the final computed value of $J\left(\beta_{n}\right)$. We point out the asymptotic superlinear decay as $\beta \rightarrow 0$. 


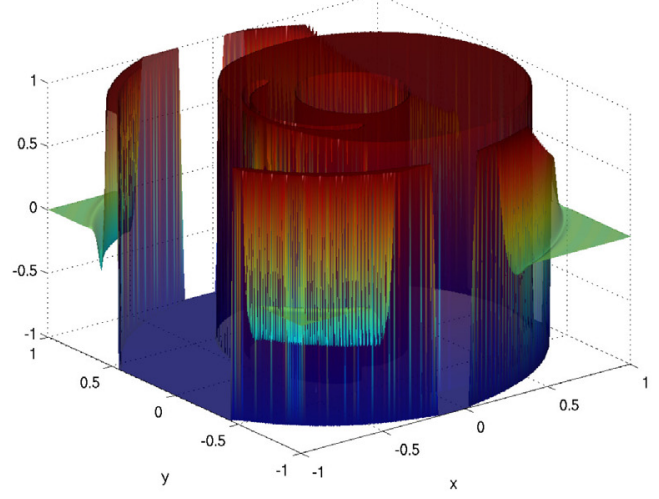

(A) $u_{\alpha}, \alpha=5 \times 10^{-3}$

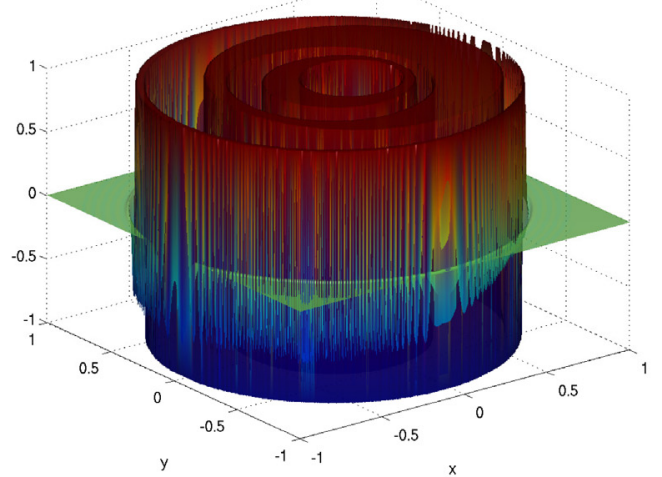

(c) $u_{\alpha}, \alpha=5 \times 10^{-4}$

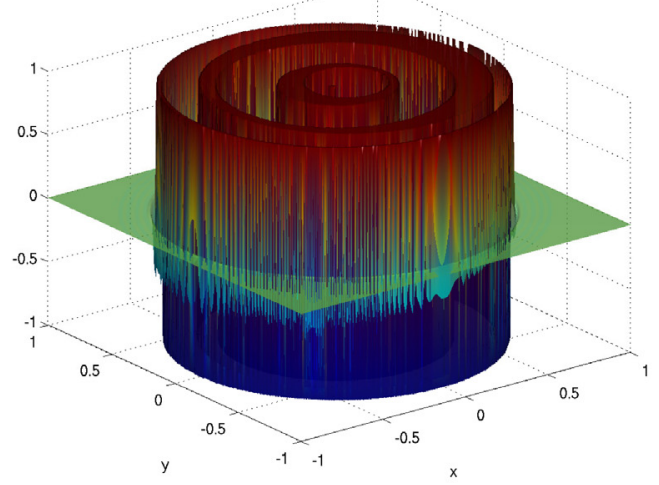

(E) $u_{\alpha}, \alpha=5 \times 10^{-5}$

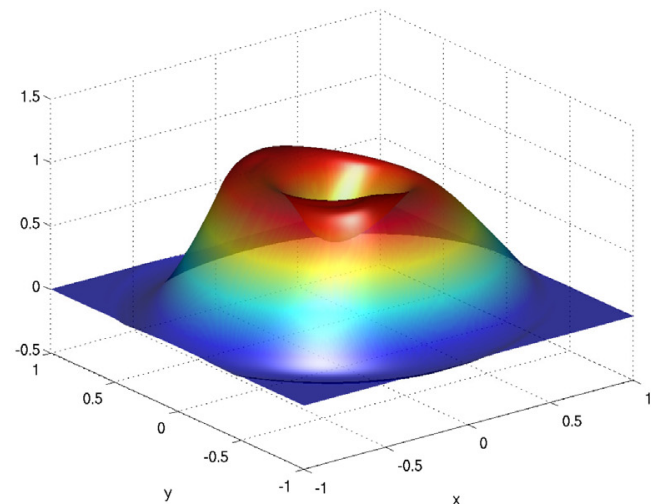

(в) $y_{\alpha}, \alpha=5 \times 10^{-3}$

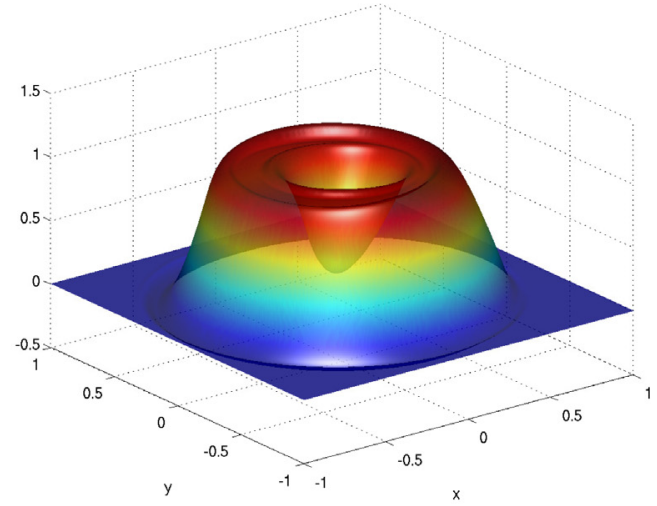

(D) $y_{\alpha}, \alpha=5 \times 10^{-4}$

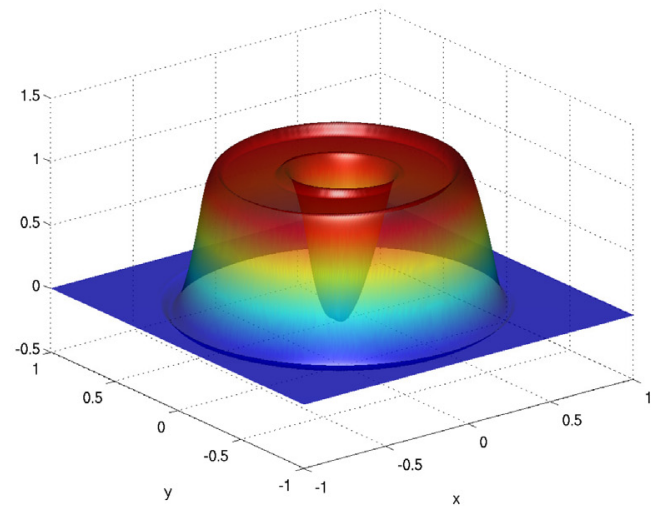

(F) $y_{\alpha}, \alpha=5 \times 10^{-5}$

FiguRE 2. Optimal controls $u_{\alpha}$ and states $y_{\alpha}$ for the target $z_{1}$ and different $\alpha$. 


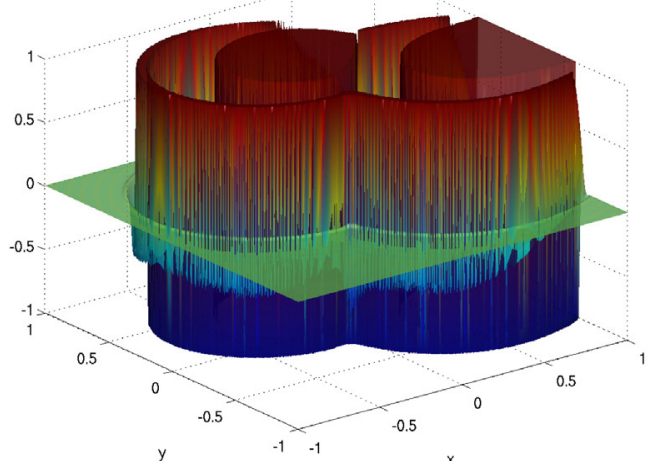

(A) $u_{\alpha}, \alpha=5 \times 10^{-3}$

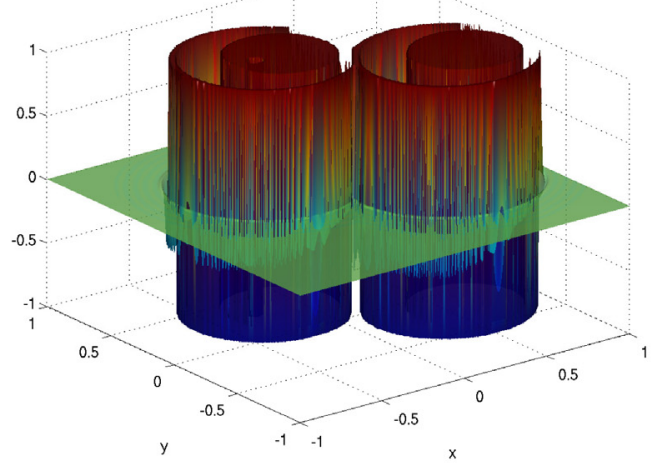

(C) $u_{\alpha}, \alpha=5 \times 10^{-4}$

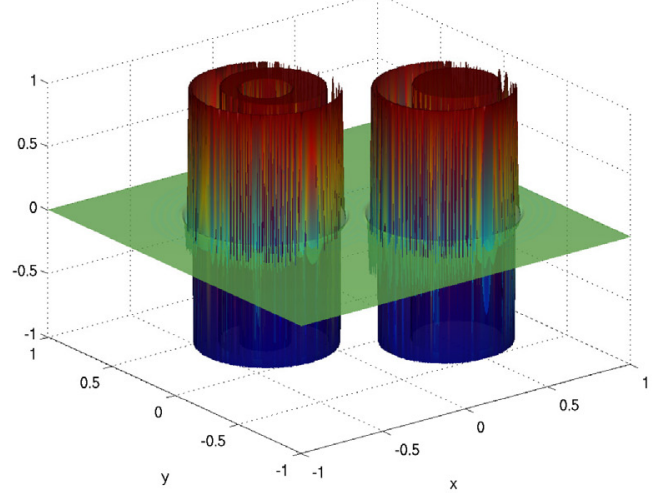

(E) $u_{\alpha}, \alpha=5 \times 10^{-5}$

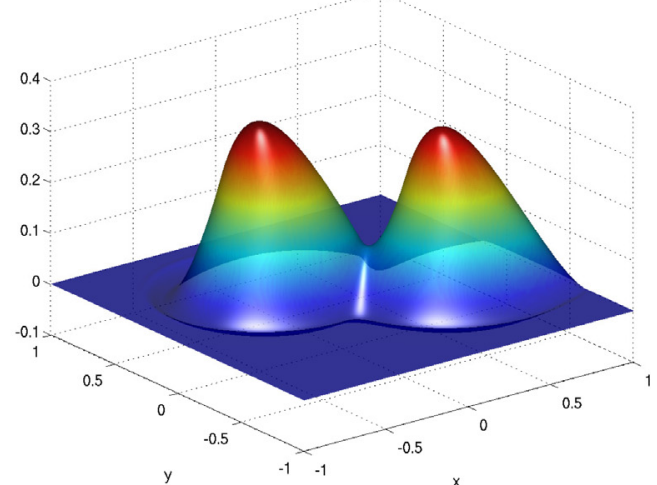

(в) $y_{\alpha}, \alpha=5 \times 10^{-3}$

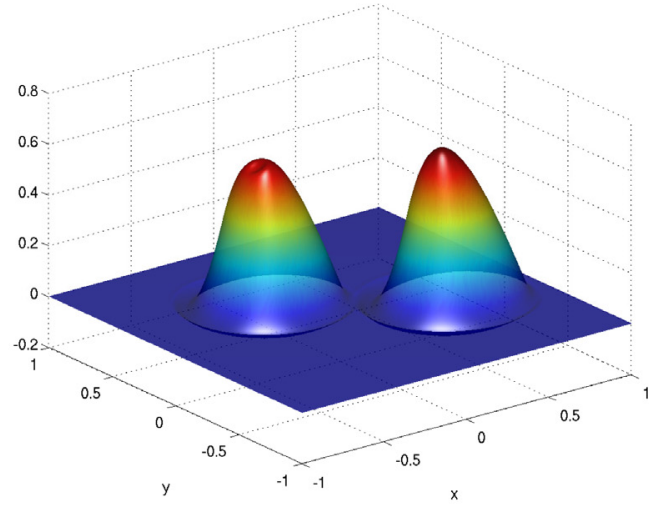

(D) $y_{\alpha}, \alpha=5 \times 10^{-4}$

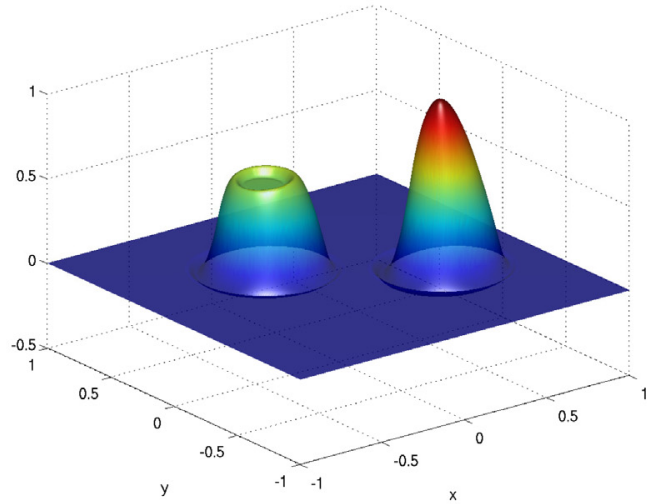

(F) $y_{\alpha}, \alpha=5 \times 10^{-5}$

FiguRE 3. Optimal controls $u_{\alpha}$ and states $y_{\alpha}$ for the target $z_{2}$ and different $\alpha$. 


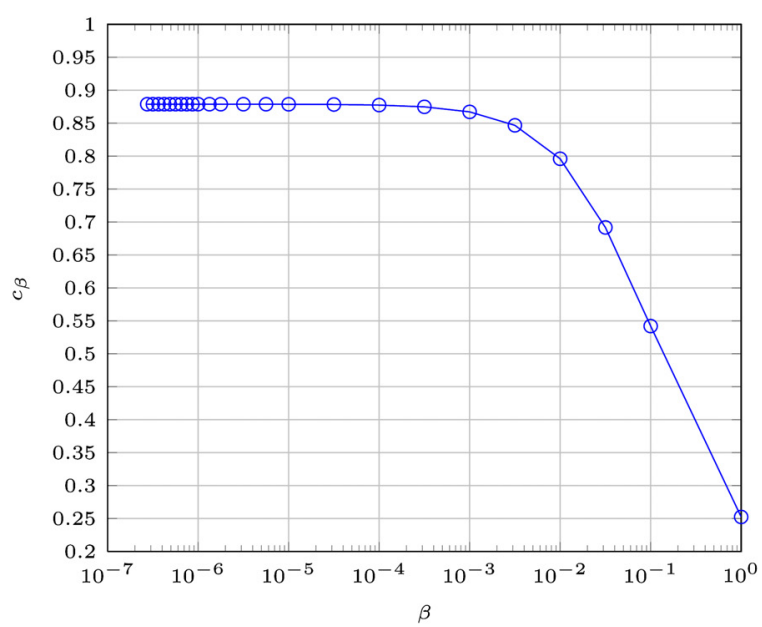

(A) optimal $\mathrm{L}^{\infty}$ bound $c_{\beta}$

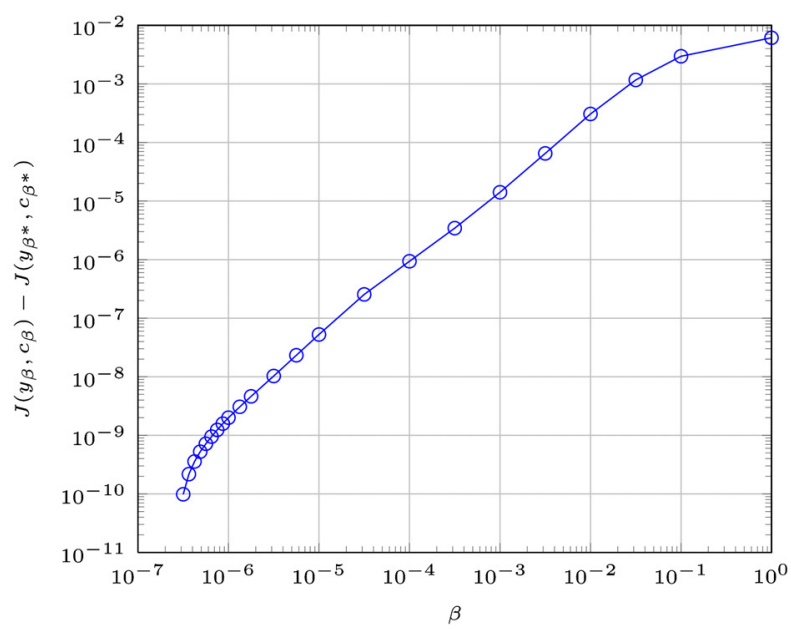

(B) difference $J\left(y_{\beta}, c_{\beta}\right)-J\left(y_{\beta_{\min }}, c_{\beta_{\min }}\right)$

FIGURE 4. Illustration of the convergence behavior with respect to $\beta$. Every circle corresponds to a computed step in the continuation method.

TABLE 2. Convergence of semi-smooth Newton method. Shown is the norm of the residual of (4.1) for the iterates $\left(p^{k}, c^{k}\right)$.

\begin{tabular}{cccccccccc}
\hline$k$ & 0 & 1 & 2 & 3 & 4 & 5 & 6 & 7 & 8 \\
\hline$\left\|T\left(p^{k}, c^{k}\right)\right\|_{\mathrm{L}^{2}}$ & 174 & 6.75 & 1.93 & 0.584 & 0.197 & 0.0224 & $2.36 \times 10^{-4}$ & $1.62 \times 10^{-7}$ & $1.12 \times 10^{-10}$ \\
\hline
\end{tabular}

We indicate the superlinear convergence of the semi-smooth Newton method by fixing $\beta=5 \times 10^{-2}$ and computing $p_{\beta}, c_{\beta}$ from the starting guess $\left(p_{0}, c_{0}\right)=(0,0)$ (again, for target $z_{2}$ and $\left.\alpha=5 \times 10^{-3}\right)$. Table 2 shows the norm of the residual $\left\|T\left(p^{k}, c^{k}\right)\right\|_{\mathrm{L}^{2}}$ in the semi-smooth Newton method, verifying the locally superlinear convergence shown in Theorem 4.2.

Finally, we consider the effect of the geometry of the control domain on the optimal control and state. For this, we choose the target $z_{3}(x, y)=1$, set $b=(0,0)$ and compare subdomains of equal area on which the control is allowed to act ( $c f$. Rem. 3.3): The control domain $\omega_{n}$ consists of 1,4 or 9 uniformly distributed squares whose areas each sum to 1 (see Fig. 5). The penalty parameter was fixed at $\alpha=10^{-3}$, and to allow quantitative comparison, the continuation in $\beta$ was terminated in each case when $\beta \leq 10^{-7}$ was satisfied. The resulting controls and states are shown in Figure 5, and the corresponding optimal $\mathrm{L}^{\infty}$-bound $c^{*}$ are 4.1039, 4.3707 and 4.6298 , respectively.

\section{CONCLUSiOn}

A semi-smooth Newton technique based on an appropriate regularization was analyzed and investigated numerically for a class of minimum effort optimal control problems for elliptic equations. The numerical results show that while the unregularized minimum effort controls can be expected to be of bang-bang type, the regularized controls mostly assume values on the boundary of the admissible control set and are zero on open subsets of the control domain. This sparsity property could be of practical interest in itself, and can certainly be the focus of further investigations. 


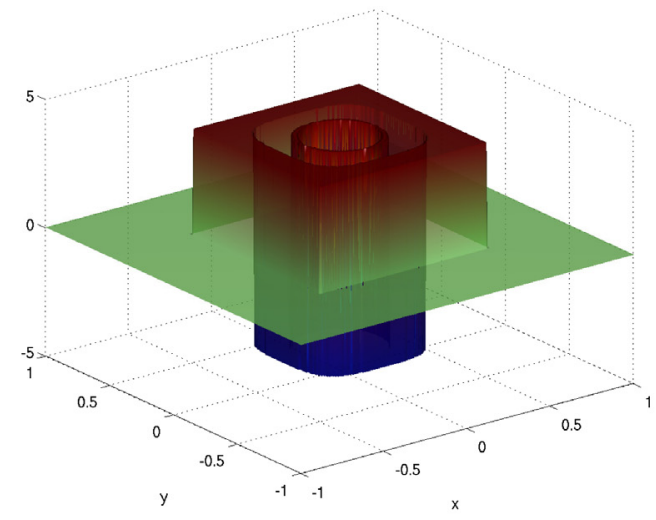

(A) optimal control $(c u)$ for control domain $\omega_{1}$

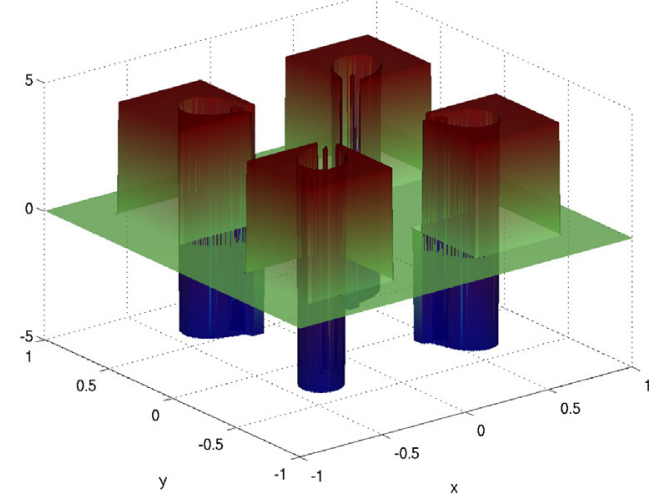

(C) optimal control (cu) for control domain $\omega_{2}$

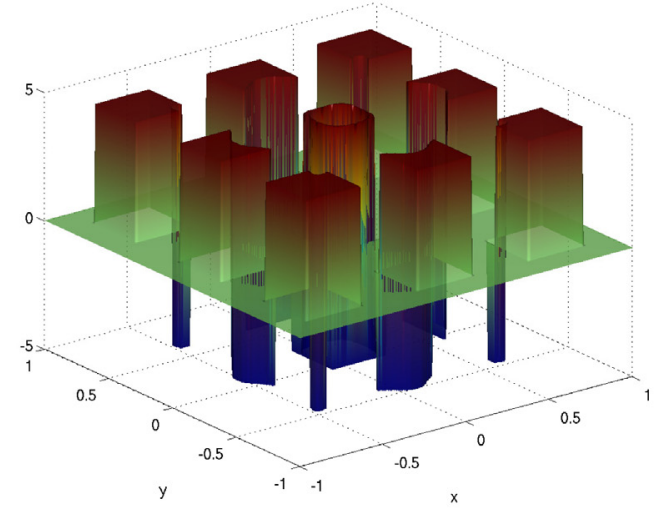

(E) optimal control $(\mathrm{cu})$ for control domain $\omega_{3}$

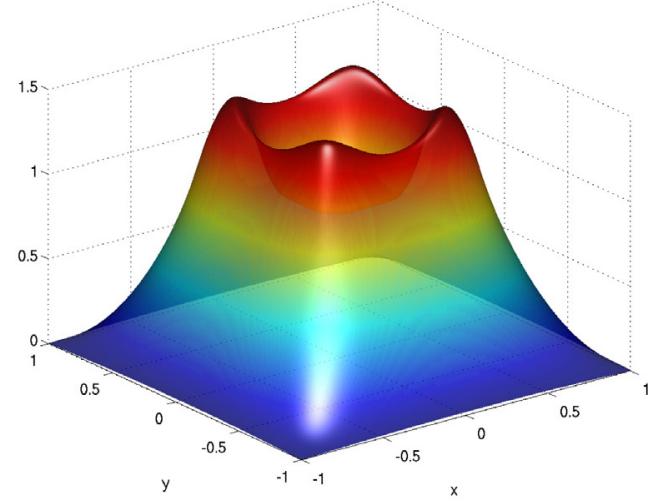

(в) optimal state $y$ for control domain $\omega_{1}$

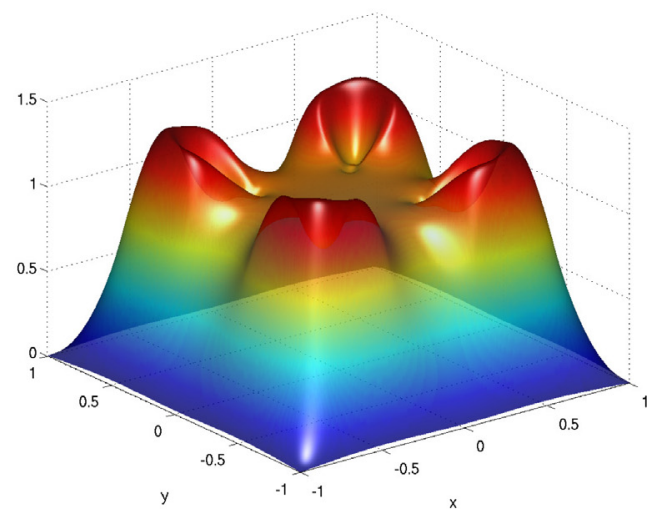

(D) optimal state $y$ for control domain $\omega_{2}$

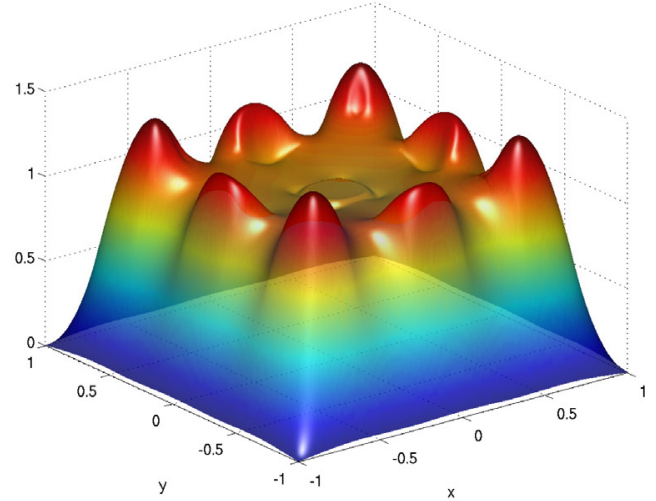

(F) optimal state $y$ for control domain $\omega_{3}$

Figure 5. Optimal controls $(c u)$ and states $y$ for the target $z_{3}=1$ and different control domains $\left(\alpha=10^{-3}\right)$. 
Acknowledgements. The research of the first and last named authors was supported in part by the Austrian Science Fund (FWF) under grant SFB F32 (SFB "Mathematical Optimization and Applications in Biomedical Sciences"). The research of the second named author was partially supported by the Army Research Office under DAAD19-02-1-0394.

\section{Appendix A. Proof of Proposition 3.1}

It will be convenient to introduce the reduced cost

$$
F(u, c)=\frac{1}{2}\left\|A^{-1}(c u)-z\right\|_{\mathrm{L}^{2}}^{2}+\frac{\beta c}{2}\|u\|_{\mathrm{L}^{2}}^{2}+\frac{\alpha}{2} c^{2},
$$

with the corresponding optimality conditions

$$
\left\{\begin{array}{l}
c_{\beta}\left\langle\beta u_{\beta}-A^{-*} z+c_{\beta} A^{-*} A^{-1} u_{\beta}, u-u_{\beta}\right\rangle_{\mathrm{L}^{2}} \geq 0 \quad \text { for all }\|u\|_{\mathrm{L}^{\infty}} \leq 1, \\
\alpha c_{\beta}+\frac{\beta}{2}\left\|u_{\beta}\right\|_{\mathrm{L}^{2}}^{2}-\left\langle u_{\beta}, A^{-*} z\right\rangle_{\mathrm{L}^{2}}+c_{\beta}\left\|A^{-1} u_{\beta}\right\|_{\mathrm{L}^{2}}^{2}=0 .
\end{array}\right.
$$

To apply a Taylor expansion of $F$, we compute the partial derivatives of $F$ at $\left(u_{\beta}, c_{\beta}\right)$ :

$$
\begin{aligned}
& F_{u u}=c_{\beta}^{2} A^{-*} A^{-1}+\beta c_{\beta} I, \quad F_{c c}=\left\|A^{-1} u_{\beta}\right\|_{L^{2}}+\alpha, \quad F_{u c}=2 c_{\beta} A^{-*} A^{-1} u_{\beta}-A^{-*} z+\beta u_{\beta}, \\
& F_{u u c}=2 c_{\beta} A^{-*} A^{-1}+\beta I, \quad F_{c c u}=2 A^{-*} A^{-1} u_{\beta}, \quad F_{u u c c}=2 A^{-*} A^{-1},
\end{aligned}
$$

with $F_{c c c}, F_{u u u}$, the remaining fourth-order and all higher-order derivatives being zero.

Now let $(u, c)$ be any admissible pair and set

$$
\hat{u}=u-u_{\beta}, \quad \hat{c}=c-c_{\beta} .
$$

Applying Taylor expansion of $F(u, c)$ at $\left(u_{\beta}, c_{\beta}\right)$ and making use of (A.1), we find that

$$
\begin{aligned}
F(u, c)-F\left(u_{\beta}, c_{\beta}\right)= & c_{\beta}\left\langle\beta u_{\beta}-A^{-*} z+c_{\beta} A^{-*} A^{-1} u_{\beta}, \hat{u}\right\rangle_{\mathrm{L}^{2}}+\frac{c_{\beta}^{2}}{2}\left\|A^{-1} \hat{u}\right\|_{\mathrm{L}^{2}}^{2}+\frac{\beta c_{\beta}}{2}\|\hat{u}\|_{\mathrm{L}^{2}}^{2} \\
& +\frac{1}{2}\left\|A^{-1} u_{\beta}\right\|_{\mathrm{L}^{2}}^{2}+\alpha \hat{c}^{2}+\left\langle\beta u_{\beta}-A^{-*} z+c_{\beta} A^{-*} A^{-1} u_{\beta}, \hat{u}\right\rangle_{\mathrm{L}^{2}} \hat{c} \\
& +c_{\beta}\left\langle A^{-*} A^{-1} u_{\beta}, \hat{u}\right\rangle_{\mathrm{L}^{2}} \hat{c}+c_{\beta}\left\|A^{-1} \hat{u}\right\|_{\mathrm{L}^{2}}^{2} \hat{c}+\left\langle A^{-1} u_{\beta}, A^{-1} \hat{u}\right\rangle_{\mathrm{L}^{2}} \hat{c}^{2}+\frac{1}{2}\left\|A^{-1} \hat{u}\right\|_{\mathrm{L}^{2}}^{2} \hat{c}^{2} \\
\geq & \frac{c_{\beta}^{2}}{2}\left\|A^{-1} \hat{u}\right\|_{\mathrm{L}^{2}}^{2}+\frac{\beta}{2}\left(c_{\beta}+\hat{c}\right)\|\hat{u}\|_{\mathrm{L}^{2}}^{2}+\frac{1}{2}\left(\left\|A^{-1} u_{\beta}\right\|_{\mathrm{L}^{2}}^{2}+\alpha\right) \hat{c}^{2}+c_{\beta}\left\langle A^{-1} u_{\beta}, A^{-1} \hat{u}\right\rangle_{\mathrm{L}^{2}} \hat{c} \\
& +c_{\beta}\left\|A^{-1} \hat{u}\right\|_{\mathrm{L}^{2}}^{2} \hat{c}+\left\langle A^{-1} u_{\beta}, A^{-1} \hat{u}\right\rangle_{\mathrm{L}^{2}} \hat{c}^{2}+\frac{1}{2}\left\|A^{-1} \hat{u}\right\|_{\mathrm{L}^{2}}^{2} \hat{c}^{2} \\
\geq & \frac{c_{\beta}^{2}}{2}\left(1-\frac{1}{\eta}\right)\left\|A^{-1} \hat{u}\right\|_{\mathrm{L}^{2}}^{2}+\frac{\beta}{2}\left(c_{\beta}+\hat{c}\right)\|\hat{u}\|_{\mathrm{L}^{2}}^{2}+\frac{1}{2}\left(\alpha-\eta\left\|A^{-1} u_{\beta}\right\|_{\mathrm{L}^{2}}^{2}\right) \hat{c}^{2},
\end{aligned}
$$

where we have used that

$$
\left\langle A^{-1} u_{\beta}, A^{-1} \hat{u}\right\rangle_{\mathrm{L}^{2}}=\left\langle\sqrt{\eta} A^{-1} u_{\beta},(\sqrt{\eta})^{-1} A^{-1} \hat{u}\right\rangle_{\mathrm{L}^{2}} \geq-\frac{\eta}{2}\left\|A^{-1} u_{\beta}\right\|_{\mathrm{L}^{2}}^{2}-\frac{1}{2 \eta}\left\|A^{-1} \hat{u}\right\|_{\mathrm{L}^{2}}^{2}
$$

for every $\eta>0$.

Let $K=\sup \left\{\left\|A^{-1} u\right\|_{\mathrm{L}^{2}}^{2}:\|u\|_{\mathrm{L}^{\infty}} \leq 1\right\}$. Then the factors in front of $\left\|A^{-1} \hat{u}\right\|_{\mathrm{L}^{2}}^{2}$ and $\hat{c}^{2}$ are nonnegative if $\eta=1$ and $\alpha>K^{2}$ holds. Under this condition, we have that

$$
F(u, c)-F\left(u_{\beta}, c_{\beta}\right) \geq \frac{\beta}{2}\left(c_{\beta}+\hat{c}\right)\|\hat{u}\|_{\mathrm{L}^{2}}^{2}+\left(\alpha-K^{2}\right) \hat{c}^{2}+c_{\beta}\left\|A^{-1} \hat{u}\right\|_{\mathrm{L}^{2}}^{2} \hat{c} .
$$

Now for fixed $\beta>0$, let $\left(u_{\beta}, c_{\beta}\right)$ and $\left(u_{\beta}^{\prime}, c_{\beta}^{\prime}\right)$ be two solutions to $\left(\mathcal{P}_{\beta}\right)$. Without loss of generality, we may assume that $c_{\beta}^{\prime} \geq c_{\beta}$. Taking $(u, c)=\left(u_{\beta}^{\prime}, c_{\beta}^{\prime}\right)$, we deduce from (A.3) that $\left(u_{\beta}, c_{\beta}\right)=\left(u_{\beta}^{\prime}, c_{\beta}^{\prime}\right)$. Moreover, if for two possibly different solutions we have $c_{\beta}=c_{\beta}^{\prime}$, then from (A.2) with any $\eta>1$ we find $u_{\beta}=u_{\beta}^{\prime}$. Conversely, if $u_{\beta}=u_{\beta}^{\prime}$ holds, then by choosing $0<\eta<\frac{\alpha}{K}$ we obtain $c_{\beta}=c_{\beta}^{\prime}$. 


\section{Appendix B. Comparison of Regularizations}

In this section we compare the chosen regularization strategy, where the penalty term is scaled linearly with $c$, with two alternatives where the penalty term is constant or quadratic in $c$. We restrict the discussion to the case $c>0$. First, we consider the regularization

$$
\left\{\begin{array}{l}
\min _{c \in \mathbb{R}_{+}, u \in \mathrm{L}^{\infty}} \frac{1}{2}\|y-z\|_{\mathrm{L}^{2}}^{2}+\frac{\beta}{2}\|u\|_{\mathrm{L}^{2}}^{2}+\frac{\alpha}{2} c^{2} \\
\text { s.t. } A y=c u \quad \text { in } \Omega \\
\|u\|_{\mathrm{L}^{\infty}} \leq 1 .
\end{array}\right.
$$

The corresponding optimality system is

$$
\left\{\begin{array}{l}
0 \in \beta u-c p+\partial I_{\left\{\|u\|_{L^{\infty}} \leq 1\right\}} \\
0=\alpha c-\langle u, p\rangle \\
0=y-z+A^{*} p \\
0=A y-c u
\end{array}\right.
$$

Again, we can rewrite the first two relations by pointwise inspection as

$$
u(x)=\operatorname{sign}_{\beta}(c p)(x)= \begin{cases}1 & \text { if } c p(x)>\beta \\ -1 & \text { if } c p(x)<-\beta \\ \frac{1}{\beta} c p & \text { if }|c p(x)| \leq \beta\end{cases}
$$

and

to obtain the reduced system

$$
\alpha c=\frac{1}{c}\|c p\|_{\beta}:=\int_{\{|c p|>\beta\}}|p(x)| \mathrm{d} x+\frac{c}{\beta} \int_{\{|c p| \leq \beta\}}|p(x)|^{2} \mathrm{~d} x
$$

$$
\left\{\begin{array}{l}
A A^{*} p+c \operatorname{sign}_{\beta}(c p)=A z \\
\alpha c-\frac{1}{c}\|c p\|_{\beta}=0
\end{array}\right.
$$

Observe that now we have the product of $c$ and $p$ in the smoothed terms. If we formally compute the semi-smooth Newton step by fixing one variable and differentiating case by case, we obtain (setting $\mathcal{A}_{+}=\{x \in \Omega: c p(x)>\beta\}$ and so on) the system

$$
\left\{\begin{array}{l}
A A^{*} \delta p+c^{2} \frac{1}{\beta} \chi_{\mathcal{I}} \delta p+\left(\chi_{\mathcal{A}_{+}}-\chi_{\mathcal{A}_{-}}+\frac{2}{\beta} c^{k} p^{k}\right) \delta c=-\left(A A^{*} p^{k}+c \operatorname{sign}_{\beta}\left(p^{k}\right)-A z\right), \\
\alpha \delta c-\left\langle\left(\chi_{\mathcal{A}_{+}}-\chi_{\mathcal{A}_{-}}+\frac{2}{\beta} c^{k} p^{k}\right), \delta p\right\rangle-\frac{1}{\beta}\left\|p^{k} \chi_{\mathcal{I}}\right\|_{\mathrm{L}^{2}} \delta c=-\left(\alpha c-\left\|p^{k}\right\|_{\mathrm{L}_{\beta}^{1}}\right) .
\end{array}\right.
$$

To show that this defines a positive definite operator, we would now need to argue that the term $\left(\alpha-\frac{1}{\beta}\left\|p^{k} \chi_{\mathcal{I}}\right\|_{\mathrm{L}^{2}}^{2}\right)$ is positive.

On the other hand, we can regularize with the scaled control $c u$, which leads to

$$
\left\{\begin{array}{l}
\min _{c \in \mathbb{R}_{+}, u \in \mathrm{L}^{\infty}} \frac{1}{2}\|y-z\|_{\mathrm{L}^{2}}^{2}+\frac{\beta}{2}\|c u\|_{\mathrm{L}^{2}}^{2}+\frac{\alpha}{2} c^{2} \\
\text { s.t. } A y=c u \quad \text { in } \Omega, \\
\|u\|_{\mathrm{L}^{\infty}} \leq 1 .
\end{array}\right.
$$

The corresponding optimality system is

$$
\left\{\begin{array}{l}
0 \in \beta c^{2} u-c p+\partial I_{\left\{\|u\|_{L^{\infty}} \leq 1\right\}} \\
0=\alpha c+\beta c\|u\|_{L^{2}}^{2}-\langle u, p\rangle \\
0=y-z+A^{*} p \\
0=A y-c u
\end{array}\right.
$$


Pointwise inspection then allows expressing the first two relations as

$$
u(x)=\operatorname{sign}_{\beta c}(p)(x)= \begin{cases}1 & \text { if } p(x)>\beta c \\ -1 & \text { if } p(x)<-\beta c \\ \frac{1}{\beta c} p(x) & \text { if }|p(x)| \leq \beta c\end{cases}
$$

and

$$
\alpha c=\int_{\{|p|>\beta c\}}(|p(x)|-\beta c) \mathrm{d} x .
$$

The reduced optimality system is thus

$$
\left\{\begin{array}{l}
A A^{*} p+c \operatorname{sign}_{\beta c}(p)=A z, \\
\alpha c-\int_{\{|p|>\beta c\}}(|p(x)|-\beta c) \mathrm{d} x=0 .
\end{array}\right.
$$

Here, we have $c$ appearing in the definition of the smoothed functions.

\section{REFERENCES}

[1] J.Z. Ben-Asher, E.M. Cliff and J.A. Burns, Computational methods for the minimum effort problem with applications to spacecraft rotational maneuvers, in IEEE Conf. on Control and Applications (1989) 472-478.

[2] C. Clason, K. Ito and K. Kunisch, Minimal invasion: An optimal L ${ }^{\infty}$ state constraint problem. ESAIM: M2AN 45 (2010) $505-522$.

[3] I. Ekeland and R. Témam, Convex analysis and variational problems, Society for Industrial and Applied Mathematics, Philadelphia, PA, USA (1999).

[4] D. Gilbarg and N.S. Trudinger, Elliptic Partial Differential Equations of Second Order, Classics in Mathematics. SpringerVerlag, Berlin (2001). Reprint of the 1998 edition.

[5] T. Grund and A. Rösch, Optimal control of a linear elliptic equation with a supremum norm functional. Optim. Methods Softw. 15 (2001) 299-329.

[6] M. Gugat and G. Leugering, $L^{\infty}$-norm minimal control of the wave equation: On the weakness of the bang-bang principle. ESAIM Control Optim. Calc. Var. 14 (2008) 254-283.

[7] K. Ito and K. Kunisch, Lagrange Multiplier Approach to Variational Problems and Applications, Advances in Design and Control. Society for Industrial and Applied Mathematics (SIAM), Philadelphia, PA 15 (2008).

[8] K. Ito and K. Kunisch, Minimal effort problems and their treatment by semismooth newton methods. SIAM J. Control Optim. 49 (2011) 2083-2100.

[9] O.A. Ladyzhenskaya and N.N. Ural'tseva, Linear and quasilinear elliptic equations. Translated from the Russian by Scripta Technica, Inc. Translation, edited by L. Ehrenpreis, Academic Press, New York (1968).

[10] L.W. Neustadt, Minimum effort control systems. SIAM J. Control Ser. A 1 (1962) 16-31.

[11] U. Prüfert and A. Schiela, The minimization of a maximum-norm functional subject to an elliptic PDE and state constraints. Z. Angew. Math. Mech. 89 (2009) 536-551.

[12] A. Schiela, A simplified approach to semismooth Newton methods in function space. SIAM J. Optim. 19 (2008) $1417-1432$.

[13] Z. Sun and J. Zeng, A damped semismooth Newton method for mixed linear complementarity problems. Optim. Methods Softw. 26 (2010) 187-205.

[14] G.M. Troianiello, Elliptic Differential Equations and Obstacle Problems. The University Series in Mathematics, Plenum Press, New York (1987).

[15] F. Tröltzsch, Optimal Control of Partial Differential Equations: Theory, Methods and Applications. American Mathematical Society, Providence (2010). Translated from the German by Jürgen Sprekels.

[16] E. Zuazua, Controllability and observability of partial differential equations: some results and open problems, in Handbook of differential equations: evolutionary equations. Handb. Differ. Equ., Elsevier, North, Holland, Amsterdam III (2007) 527-621. 